\section{EXCHANGE YOUR STOCK AND SAVE}

When technology changes so fast, it can be hard to keep up with the costs, and even harder to dispose of surplus or redundant stock.

If you have a stock room overflowing with excess items but have no idea how to dispose of them without leaving them to the mercy of the landfill, visit the Dental Stock X-Change, the UK's first online market place to cater exclusively to the dental industry.

General and veterinary dentists, nurses, students and technicians can all benefit from using the Dental Stock

\section{SUPERIOR VISION AND COMFORT}

Blackwell Supplies offer clinicians a wide selection of enhanced magnification equipment, including the Hogies range of operating loupes.

Hogies MiniScope flip-up surgical loupes offer newly-qualified dentists a cost-effective entry into the world of loupes, enabling more accurate diagnoses, whilst reducing operator stress. Made of multi-coated glass and providing either $2.5 x$ or $3.0 \times$ magnification with a working distance of $420 \mathrm{~mm}$, Hogies MiniScope loupes are fully adjustable, allowing for the fine-tuning of interpupillary distance and declination angle to suit every clinical situation, as well as the optical needs of most practitioners.

Each set of Hogies MiniScope loupes is supported by the Hogies Nova
X-Change to buy and sell dental products and equipment. Here you can search easily for relevant items without having to go through page after page of non-dental stock. Not only could you find a bargain, but you can also do your bit for the environment, by effectively recycling your old equipment and redundant materials.

By buying and selling through the Dental Stock X-Change, you can make a return on your old items, purchase top quality equipment at a fraction of the retail price, and ensure that your unwanted stock finds a good home.

Reader response number 60 companion frame, which can be further enhanced with a range of magnetically attachable accessories, providing added flexibility. These include: LED Headlight; Laser Shield; Polycarbonate Protection Shield; and Rimless Prescription Adaptor.

Available in a choice of six fashionable colours, and weighing in at a lightweight $48 \mathrm{~g}$, dental professionals can be assured of superior vision as well as an attractive combination of style and comfort during clinical procedures.

Reader response number 61

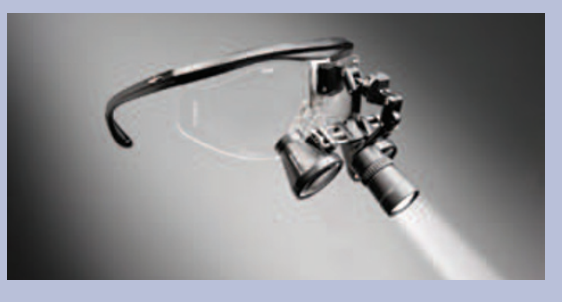

PROJECT AN INTEGRATED BRAND IDENTITY

Leading British manufacturer of specialist treatment couches and chairs, Plinth 2000, is offering the option of screen printing logos, crests, club badges and other designs onto its vinyl upholstery, across the complete product range. Single-colour images can now be printed in any Pantone shade onto any choice of upholstery, whilst two-, threeand four-colour reproduction will be offered if there is customer demand. The price for a one-off order is just £75 excluding VAT, including set-up and screen origination, with initial run-ons at $£ 55$ and further volume discounts available.

According to Plinth 2000, branding and corporate colours are increasingly important in the healthcare sector for those who wish to project an integrated brand identity. There is also a case for printing cartoon characters or other bright images in paediatric departments, to create a child-friendly look in an otherwise stressful environment.

Integrated across elements like signing, stationery, workwear and print, a consistent identity helps build brand awareness and customer loyalty; now, applied to clinical and treatment room furniture, it will add to an overall image of professionalism.

For independent clinics Plinth 2000's customising option helps give a distinctive competitive edge in a crowded marketplace.

Plinth 2000 offers a wide selection of purpose-designed treatment couches and chairs. All upholstery is vinyl, washable, fire-retardant and mostly includes patented antimicrobial technology, with a standard choice of 18 colours. Special upholstery shades are also available to order, for matching specific house colours, and, taking advantage of a manufacturer that handles all design and assembly inhouse, logos can be printed onto any product in any colour, usually on the backrest for optimum exposure.

Reader response number 63
Goldspeed handpieces is sanitation; they are not only fully autoclavable, but also include a device to repel and remove ous benefits to clinicians, including . titanium heads and handgrips for lightness and strength; high intensity fibre optic lighting for long-lasting, white illumination; compatibility with all ISO 3964 micromotor couplings; visibility for general, endodontic, restorative, prosthetic and surgical fields; and contra-angled design for manoeuvrability and balance.

For clinicians requiring the highest levels of performance, efficiency, consistency and effectiveness, Goldspeed excellence is within reach.

Reader response number 62 